\title{
Short-term relationship between solar irradiances and equatorial peak electron densities
}

\author{
X. Wang \\ University of Central Florida \\ R. Eastes \\ B. W. Reinisch \\ S. Bailey \\ C. E. Valladares
}

See next page for additional authors

Find similar works at: https://stars.library.ucf.edu/facultybib2000

University of Central Florida Libraries http://library.ucf.edu

This Article is brought to you for free and open access by the Faculty Bibliography at STARS. It has been accepted for inclusion in Faculty Bibliography 2000s by an authorized administrator of STARS. For more information, please contactSTARS@ucf.edu.

\section{Recommended Citation}

Wang, X.; Eastes, R.; Reinisch, B. W.; Bailey, S.; Valladares, C. E.; and Woods, T., "Short-term relationship between solar irradiances and equatorial peak electron densities" (2007). Faculty Bibliography 2000 s.

7765.

https://stars.library.ucf.edu/facultybib2000/7765

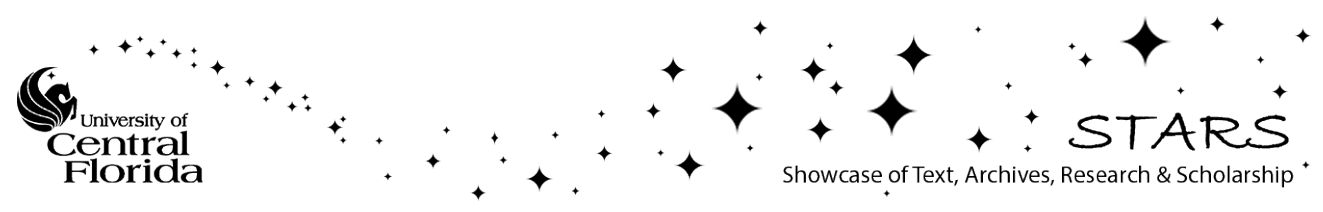


Authors

X. Wang, R. Eastes, B. W. Reinisch, S. Bailey, C. E. Valladares, and T. Woods 


\title{
Short-term relationship between solar irradiances and equatorial peak electron densities
}

\author{
X. Wang, ${ }^{1}$ R. Eastes, ${ }^{2}$ B. W. Reinisch, ${ }^{3}$ S. Bailey, ${ }^{4,5}$ C. E. Valladares, ${ }^{6}$ and T. Woods ${ }^{7}$ \\ Received 17 October 2006; revised 20 February 2007; accepted 1 March 2007; published 13 June 2007.
}

[1] The short-term relationship of the equatorial peak electron density and the solar shortwavelength irradiance is examined using foF2 observations from Jicamarca, Peru and recent solar irradiance measurements from satellites. Solar soft X-ray measurements from both the Student Nitric Oxide Explorer (SNOE) (1998-2000) and Thermosphere Ionosphere Mesosphere Energetics Dynamics (TIMED) (2002-2004) satellites as well as extreme ultraviolet (EUV) measurements from the TIMED satellite are used. Soft X-rays show similar or higher correlation with foF2 at short timescales (27 days or less) than EUV does, although the EUV correlation is higher for longer periods. For the short-term variations, both SNOE and TIMED observations have a higher correlation in the morning $(\sim 0.46)$ than in the afternoon $(\sim 0.1)$. In the afternoon, SNOE observations have a higher correlation $(\sim 0.2)$ with foF 2 than the TIMED observations $(\sim 0.1$ correlation), which may be due to differences in the solar cycle. At morning times, foF 2 has a $\sim 27$-day variation, consistent with the solar rotation rate. After noon, but not in the morning, a $\sim 13.5$-day variation consistently appears in foF2. This $\sim 13.5$-day variation is attributed to geomagnetic influences.

Citation: Wang, X., R. Eastes, B. W. Reinisch, S. Bailey, C. E. Valladares, and T. Woods (2007), Short-term relationship between solar irradiances and equatorial peak electron densities, J. Geophys. Res., 112, A06310, doi:10.1029/2006JA012128.

\section{Introduction}

[2] The ionosphere plays an important role in Earth's space weather because of the coupling processes from above and below. Consequently, understanding the causes of density variations in the ionosphere is an important but challenging problem. Forbes et al. [2000] evaluated the observed ionospheric variability and attributed it to a number of sources such as solar ionizing flux, solar wind conditions, and propagating waves: gravity waves, tides, and planetary waves. The contributions from different sources were also studied in more recent work [Mendillo et al., 2002; Altadill and Apostolov, 2003; Lastovicka et al., 2003; Pancheva and Mitchell, 2004; Fagundes et al., 2005]. Most of these studies focused on the effects of geomagnetic activity and resulting planetary waves; the contribution of solar irradiance was studied using the $F_{10.7}$ radio flux as a proxy of extreme

\footnotetext{
${ }^{1}$ Department of Electrical and Computer Engineering, University of Central Florida, Orlando, Florida, USA.

${ }^{2}$ Florida Space Institute, Kennedy Space Center, Merritt Island, Florida, USA.

${ }^{3}$ Center of Atmospheric Research, University of Massachusetts, Lowell, Massachusetts, USA.

${ }^{4}$ Geophysical Institute, University of Alaska Fairbanks, Fairbanks, Alaska, USA.

${ }^{5}$ Now at Physics Department, Virginia Tech, Blacksburg, Virginia, USA.

${ }^{6}$ Newton Resource Center, Institute for Scientific Research, Boston College, Chestnut Hill, Massachusetts, USA.

${ }^{7}$ Laboratory for Atmospheric and Space Physics, University of Colorado, Boulder, Colorado, USA.
}

Copyright 2007 by the American Geophysical Union. 0148-0227/07/2006JA012128 ultraviolet (EUV) irradiance. Although solar EUV produces most of the ionization in the $\mathrm{F}$ region, their results indicated an insignificant solar effect on the short-term variations of ionospheric densities. The use of $F_{10.7}$ as a proxy for EUV in these studies [e.g., Kane, 1992; Rishbeth, 1993; Balan et al., 1994] may have significantly affected the results.

[3] Direct measurements of solar irradiances are available from recent satellite missions. The Student Nitric Oxide Explorer (SNOE) satellite began measuring solar soft X-ray irradiances $(0.1 \mathrm{~nm}-20 \mathrm{~nm})$ in March 1998 [Bailey et al., 2000; Bailey et al., 2005]. More recently, the Thermosphere Ionosphere Mesosphere Energetics Dynamics (TIMED) satellite has measured the solar spectral irradiance $(0.1-$ $193 \mathrm{~nm}$ ) in the X-ray ultraviolet (XUV), extreme ultraviolet (EUV), and far ultraviolet (FUV) ranges from February 2002 to present [Woods et al., 2005].

[4] Recent work by Solomon et al. [2001] and Wang et al. [2006] indicate these direct measurements of the Sun's short wavelength emissions may enable us to better understand the effect of solar irradiance on the ionosphere, compared to proxies such as $F_{10.7}$. Solomon et al. [2001] studied the effects of soft X-rays on the lower ionosphere (100-200 km) and found good agreement between observed electron density profiles and model predictions when using solar irradiances from SNOE. Wang et al. [2006] found clear short-term ( $\leq 27$ days) correlations $(\sim 0.6)$ between the total electron content (TEC) and solar irradiances from SNOE, whereas these correlations are larger than those seen when using $F_{10.7}$.

[5] In order to advance the understanding of solar effects on ionospheric variations, this study examines the relation- 

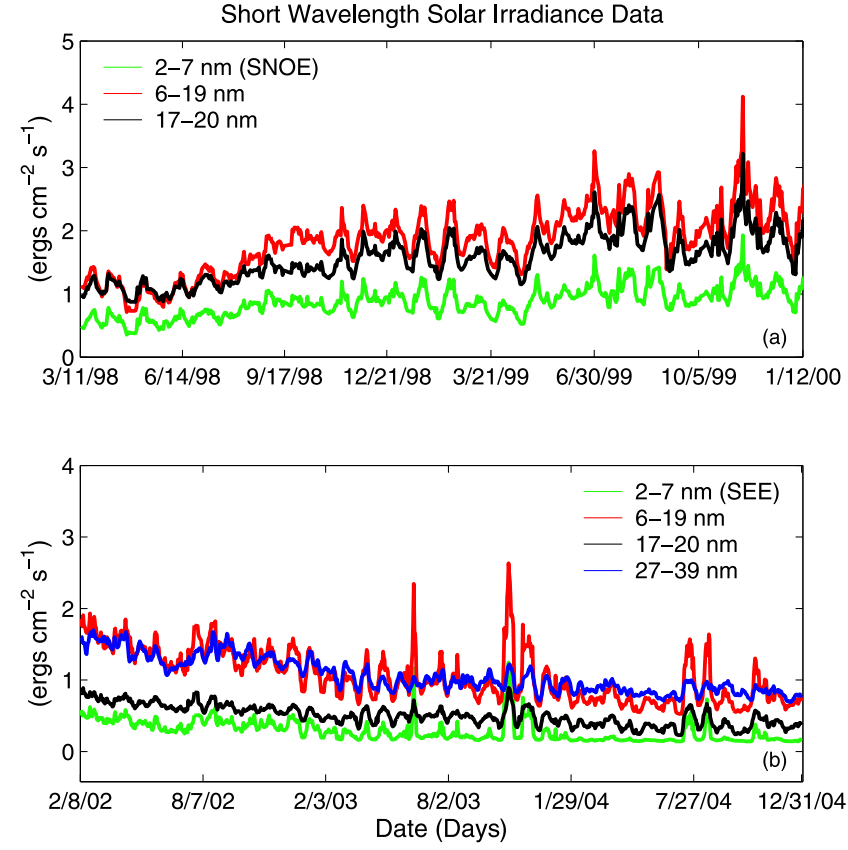

Figure 1. (a) The daily solar irradiance measured from SNOE (1998-2000). (b) The daily solar irradiance measured from TIMED (2002-2004).

ship between peak electron densities and solar irradiances (X-rays and EUV). As a measure of the peak electron densities, the critical frequencies of the F2 layer (foF2) from Jicamarca, Peru are used. The paper is presented in the following sequence: (1) data description, (2) data analysis and comparison of the short-term correlations between solar irradiances and foF2 as a function of local time, (3) discussion of how the periodic variations in foF 2 change with local time, and (4) conclusions.

\section{Data}

[6] Short-wavelength solar irradiances from SNOE and TIMED are used in this study. The SNOE satellite carried the solar X-ray photometer (SXP), which measured the solar soft X-ray irradiance in broad-wavelength bands [Bailey et al., 2000, 2005]. Each channel consisted of an X-raysensitive silicon photodiode with a thin metallic film deposited directly onto the active area. Measurements of three spectral ranges, approximately $2-7 \mathrm{~nm}, 6-19 \mathrm{~nm}$, and $17-$ $20 \mathrm{~nm}$, are used in the following analysis. The measurements used began on 11 March 1998 and continued through January 2000. The values used, shown in Figure 1a, are "daily" values. The number of solar observations each day varied from 4 (for the earliest dates) to 16; the daily average soft X-ray irradiance is calculated using the measurements without contributions from solar flares.

[7] The second set of solar data used in this study is from the Solar EUV Experiment (SEE) aboard the TIMED satellite. The SEE includes two instruments, EUV grating spectrograph (EGS) and XUV photometer system (XPS), which together measure the solar spectral irradiance from 0.1 to $193 \mathrm{~nm}$ [Woods et al., 2005]. The EGS is a normal incidence Rowland circle spectrograph with a spectral range of 27 to $193 \mathrm{~nm}$. The XPS includes nine silicon XUV photodiodes with thin film filters deposited directly on the photodiodes and measures the solar irradiance from 0.1 to $27 \mathrm{~nm}$. Between 0.1 and $27 \mathrm{~nm}$, the broad-band XPS data from SEE and a model are used to provide irradiances at 1-nm intervals. The solar irradiance measurements were retrieved from the SEE website http://see.colorado.edu/ see/. The data used in this study (version 8) include daily averages with flares removed (level 3) and data from individual orbits $(\sim 97-$ min period) with solar flares included (level 3a). The results presented in this paper are based on short-wavelength solar irradiances $(2-7,6-19$, and $17-$ $20 \mathrm{~nm}$ for the X-rays, 27-39 $\mathrm{nm}$ for the EUV), both daily averages and individual orbits, from February 2002 to December 2004. When using data from individual orbits, flares were removed by replacing data with daily averages from TIMED for which flares have been removed. The daily TIMED SEE measurements $(2-7,6-19,17-20$, and $27-39 \mathrm{~nm}$ ) are shown in Figure 1b.

[8] The foF2 data are from a Digisonde located at the Jicamarca Radio Observatory in Peru [Reinisch, 1996]. The vertical soundings of the ionosphere are normally obtained every $\sim 30 \mathrm{~min}$ and were automatically scaled using the ARTIST inversion algorithm [Reinisch and Huang, 1983]. These data are downloaded from http:/umlcar.uml.edu/ DIDBase/, provided by the Center for Atmospheric Research at the University of Massachusetts, Lowell. There are usually two to four measurements in an hour, and by averaging all measurements collected during an hour, the hourly averages are obtained. In this study, the hourly averages of foF 2 from 0700 to 1800 local time (LT) are used from March 1998 to January 2000 and from February 2002 to December 2004. An example of these data, col-
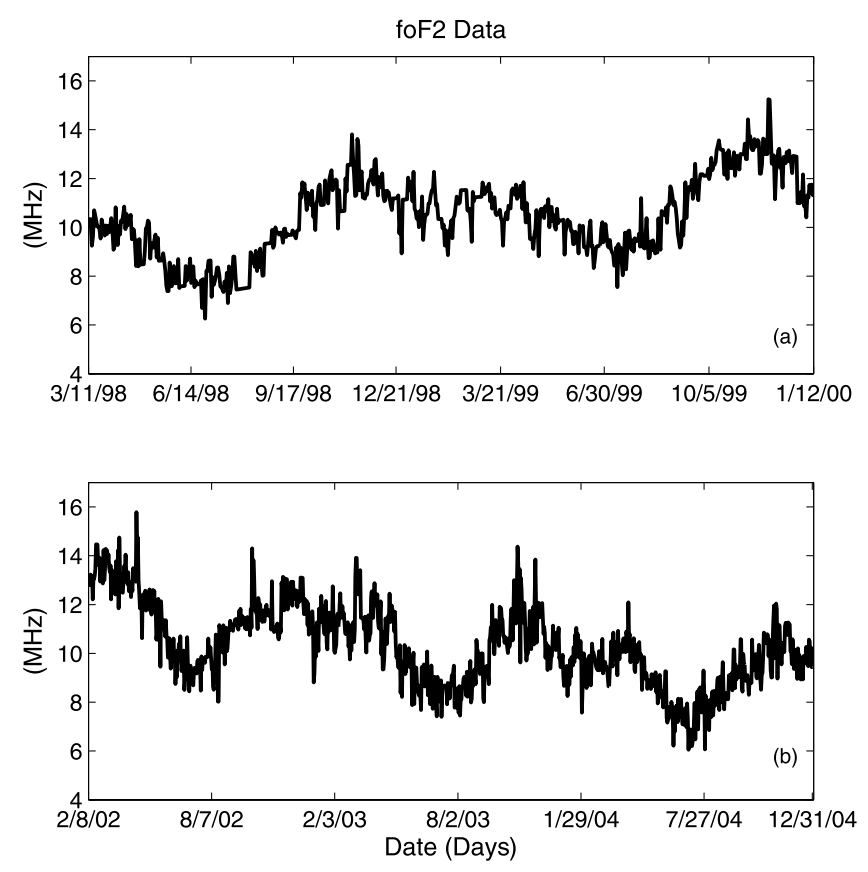

Figure 2. Averages of 0800-0900 LT measurements of foF2 from Jicamarca, Peru for (a) 1998-2000 and (b) 20022004. 
Correlations of Short-term Variations Between foF2 and Solar Data

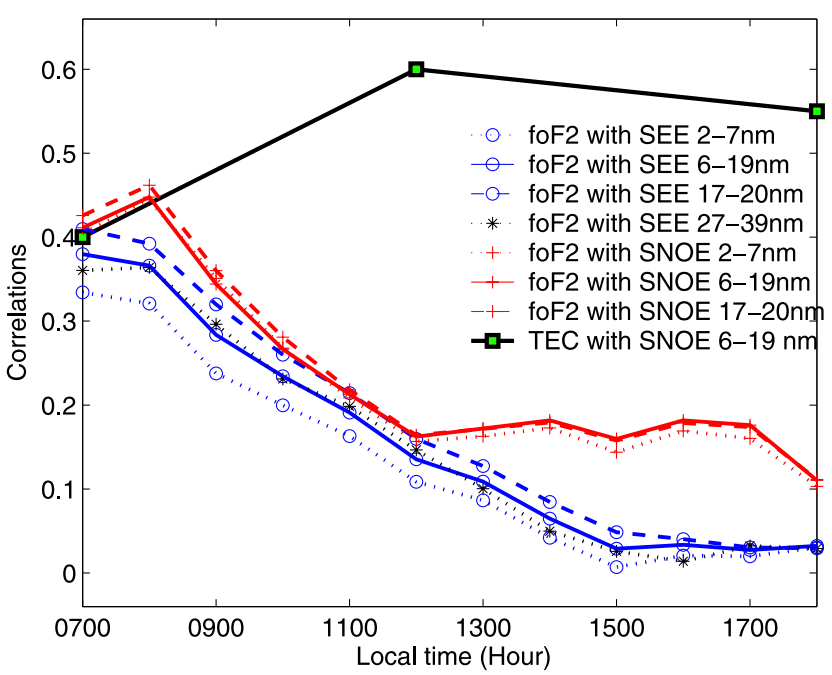

Figure 3. Correlations of short-term variations ( 27 days and less) in the daily solar irradiances and hourly foF2 data. For comparison, TEC from GPS observations are also shown, which show an opposite trend and higher correlations than foF2 with solar irradiance.

lected from 0800 to 0900 LT during 1998-2000 and 20022004, respectively, is shown in Figures $2 \mathrm{a}$ and $2 \mathrm{~b}$.

\section{Data Analysis and Correlation Discussion}

[9] In order to more easily study the short-term relationship, a 27-day moving average is removed from the solar irradiances (both daily average and individual orbits) and hourly foF 2 . The remaining signals, which will be referred to as the residuals, contain variations with periods of $\leq 27$ days. The magnitude of the correlations between the residuals is a measure of the linear, short-term relationship between solar irradiances and foF2.

[10] The correlations are similar when using solar irradiances from daily averages or individual orbits. An example, using daily solar irradiances and hourly foF2, is shown in Figure 3. It shows: (1) the foF2 has similar or higher correlations with solar soft X-rays than with solar EUV; (2) correlations between foF 2 and solar irradiance decrease from 0700 to 1800 LT; and (3) correlations in the afternoon are higher when using solar X-rays from SNOE than those from TIMED. Each of these three points will be discussed in more detail. As for the correlations between foF2 and soft X-rays, the results from using 6-19 and 17-20 $\mathrm{nm}$ are discussed since they are more significant than those from the 2-7 $\mathrm{nm}$.

[11] First, as shown in Figure 3, foF2 has similar or slightly higher correlations with soft X-rays than with EUV. For example, the foF2 (0700-0800 LT) has a 0.41 correlation with the X-rays and a 0.36 correlation with the EUV. The average difference between the correlations is 0.05 , which is approximately equal to the $95 \%$ confidence level of 0.06 . The variability of solar soft X-rays differs from that of EUV, which however does not completely determine their correlation difference (e.g., the standard deviation is 0.206 for $6-19 \mathrm{~nm}, 0.722$ for $17-20 \mathrm{~nm}$, and 0.768 for EUV; the $17-20 \mathrm{~nm}$ shows slightly higher correlation than the 6-19 $\mathrm{nm}$ with foF2 as seen in Figure 3). Neither does the normalization of these data change the results. To better understand this difference, we must first understand the relationship between the X-rays and EUV. If the X-ray and EUV irradiances were sufficiently correlated, both correlations would represent the same relationship with foF2. However, the $0.75-0.77$ correlation between the X-rays $(6-19$ and $17-20 \mathrm{~nm})$ and the EUV $(27-39 \mathrm{~nm})$ is lower than the 0.95 correlation between solar irradiances of 6-19 and 17-20 nm. Therefore it is worth considering whether X-rays and EUV are redundant.

[12] In order to better understand the differences between $\mathrm{X}$-rays and EUV, the correlation of foF2 with each, separate from the effects of the other, can be examined. This is accomplished using partial correlations [Dodge, 2006]. The correlation between foF2 and X-rays (or EUV) that remain after controlling for the EUV (or X-rays) indicates whether the additional measurement of solar irradiance provides additional information about the dependence of foF 2 on solar irradiance. Using foF 2 at 0700-0800 and 0800-0900 LT as an example, the partial correlations between foF2 and $\mathrm{X}$-rays (after isolating the EUV) are significant, 0.22 at 0700-0800 LT and 0.18 at 0800-0900 LT, compared to a $95 \%$ confidence level of 0.06 . Therefore the soft X-rays provide additional information that is useful for determining ionospheric densities.

[13] While the soft X-rays are a better short-term indicator for foF2, the EUV is a better long-term indicator according to the correlations, in Figure 4, between the raw solar and ionospheric data. Since moving averages are not subtracted, the long-term ( $>27$ days) changes are included. For example, at $0800-0900 \mathrm{LT}$, a correlation of 0.66 is seen between EUV and foF2, while the correlation between $\mathrm{X}$-rays and foF2 is 0.57 . However, short-wavelength solar irradiances generally show higher correlations with foF2 than $F_{10.7}$ does (as seen in Figure 4). This is consistent with the presence of higher correlations between TEC and X-rays than between TEC and $F_{10.7}$ [Wang et al., 2006].

[14] Second, as shown in Figure 3, correlations between foF 2 and solar irradiances decrease from 0700 to $1800 \mathrm{LT}$. For both the EUV and the X-rays, correlations decrease from 0.4 in the morning to approximately 0.1 in the afternoon. Similar decreases are seen in measurements from either SNOE or TIMED. However, the correlations between X-rays (from the SNOE) and TEC (from a Global Positioning System (GPS) receiver in Ancon, Peru [Valladares et al., 2001])

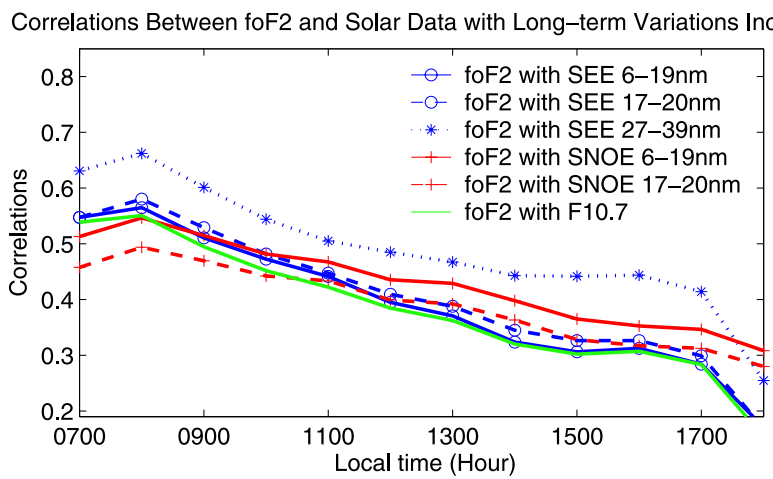

Figure 4. Correlations between solar irradiances and foF2 when the long-term ( $>27$ days) changes are included. 

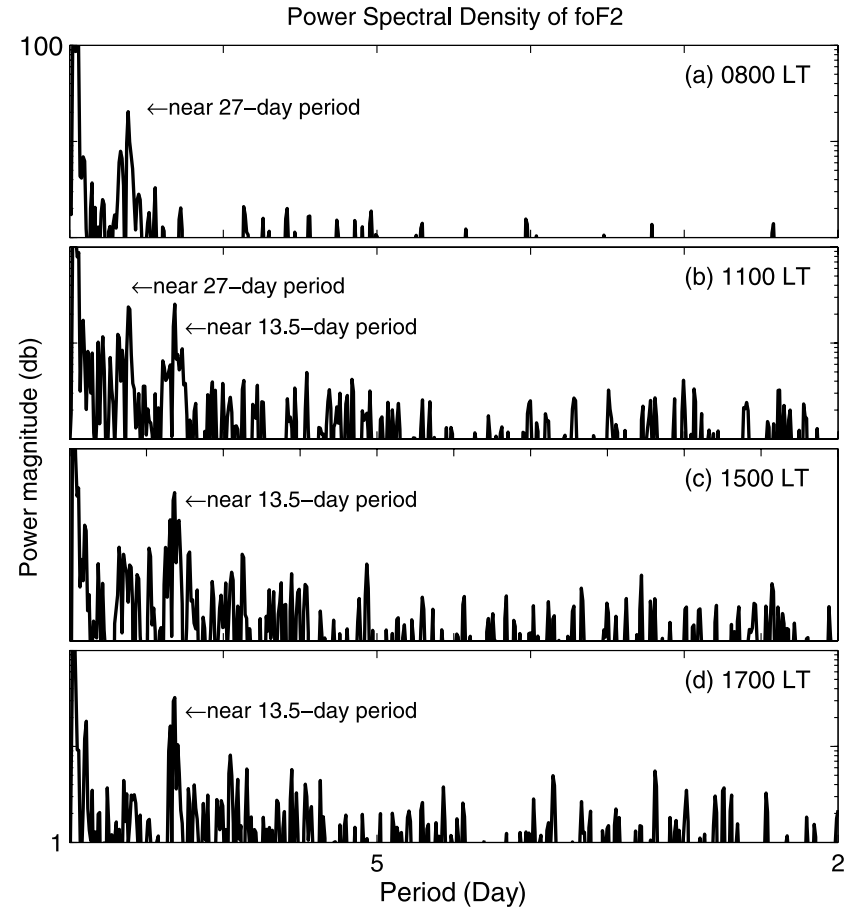

Figure 5. Power spectral density of foF 2 at (a) 0800 0900 LT, (b) 1100-1200 LT, (c) 1500-1600 LT, and (d) 1700-1800 LT. A 27-day variation is seen in the morning, and $\mathrm{a} \sim 13.5$-day variation appears near noon and persists throughout the afternoon.

increase from morning (0.4) to noon (0.6), and they do not change significantly from noon to afternoon $(0.55)$, as shown in Figure 3. This indicates TEC and foF2 respond differently to solar irradiance. The small correlations of foF2 with solar irradiances in the afternoon $(\sim 0.1)$ suggest solar effects on short-term variations are insignificant, which is consistent with previous results [Forbes et al., 2000; Lastovicka et al., 2003; Rishbeth and Mendillo, 2001]; however, early in the morning, there is a significant correlation.

[15] Third, as shown in Figure 3, correlations in the afternoon are higher when using solar X-rays from SNOE (red lines) than those from TIMED (blue lines). The difference is distinct from 1400 to $1800 \mathrm{LT}$, when correlations between foF 2 and X-rays are approximately twice as large for SNOE than for TIMED (daily averages or individual orbits). The processing algorithms for both SNOE and TIMED data are similar [Woods et al., 2005], but the data are from different parts of the solar cycle. The SNOE measurements used are from March 1998 to January 2000, the rising phase of the solar cycle with solar maximum in July 2000; whereas the TIMED measurements are from February 2002 to December 2004, the declining phase of the same solar cycle. Therefore the difference between correlations in the afternoon may be due to either a random long-term temporal variation or a variation with phase of the solar cycle.

\section{Local Time Dependence of Temporal Variations in foF2}

[16] For the foF2 observations, there are also differences in the short-term temporal variations in the morning and afternoon. Power spectra (signal strength as a function of frequency) of the solar irradiances and foF2 at different times of the day are used to understand these temporal variations.

[17] Examples of the power spectrum for foF2 are shown in Figure 5. In the upper two panels (Figures 5a and 5b), the power spectra for two times in the morning, 0800-0900 and 1100-1200 LT, are shown. Two times in the afternoon, 1500-1600 and 1700-1800 LT, are shown in the bottom two panels (Figures 5c and 5d). A $\sim 27$-day variation is seen at $0800-0900$ LT, the time when a $\sim 0.4$ correlation occurs between foF 2 and solar irradiances. This indicates the $\sim 27-$ day variation may be produced by solar irradiance variations, which is consistent with results from previous studies [e.g., Wang et al., 2006].

[18] At later times, near noon and in the afternoon, a shorter $\sim 13.5$-day period appears, and the $\sim 27$-day period fades, as seen in Figure 5. This variation seems to be related to variations in the solar wind and geomagnetic activity [Altadill and Apostolov, 2003]. In order to better understand the effects of both solar irradiance and geomagnetic activity on the variation of foF2, the power spectra for both the solar EUV irradiance and ap, a 3-hour index of geomagnetic activity, are shown in Figure 6 . The $\sim 13.5$-day variation in the solar EUV (upper panel) is hardly above the average; however, a distinct $~ 13.5$-day variation is seen in ap (bottom panel). It should be noted that the $\sim 13.5$-day variation is not always evident; it is discernable in less than half of the ap data. The power spectra are consistent with the results from Mursula and Zieger [1996]. They found that the occurrence and persistence of the 13.5-day periodicity is more prominent in the solar wind and related geomagnetic activity indices than in solar irradiances during the maximum and declining phase of solar cycles. The observations used here are also from the declining phase of the solar cycle $(2002-2004)$. Therefore the $\sim 13.5$-day period in foF2 is attributed to geomagnetic activity. The $\sim 13.5$-day period is strongest in the afternoons, when the conductivity of the F region is largest; however, changes in

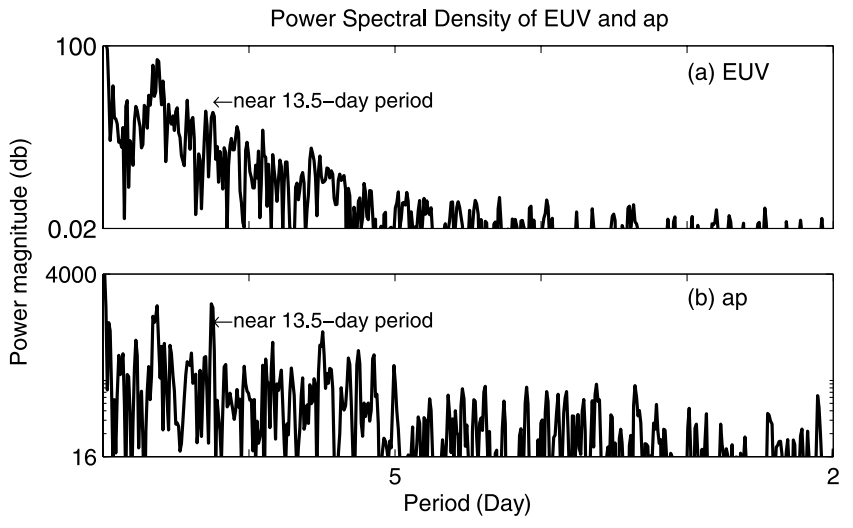

Figure 6. (a) Power spectral density of the 2002-2004 EUV measurements $(27-39 \mathrm{~nm})$ from TIMED. (b) Power spectral density of ap from 1300-1600 LT. A 13.5-day variation is distinct in the ap data but not in the solar irradiance. 
the neutral winds and electric fields may also affect the ionosphere in the afternoon.

\section{Summary}

[19] The short-term relationship of the equatorial peak electron density and the solar short-wavelength irradiance was examined using foF2 observations from Jicamarca, Peru and solar soft X-ray measurements from both the SNOE (1998-2000) and TIMED (2002-2004) satellites as well as extreme ultraviolet (EUV) measurements from the TIMED satellite. At short timescales (27 days or less), soft X-rays have a similar or slightly larger correlation with foF2 than the EUV has, but the EUV does show a higher correlation for longer periods. For the short-term $(\leq 27$ day) variations, both SNOE and TIMED observations have a higher correlation in the morning ( $\sim 0.46)$ than the afternoon $(\sim 0.1)$. In the afternoon, SNOE observations have a higher correlation $(\sim 0.2)$ with foF2 than the TIMED observations ( $\sim 0.1$ correlation). This difference in correlation may be due to either a random temporal variation or a variation with phase of the solar cycle. At morning times, foF 2 has a $\sim 27$-day variation, consistent with the solar rotation rate. In the afternoon, but not in the morning, a 13.5-day variation consistently appears in foF 2 . This $\sim 13.5$-day variation is attributed to geomagnetic influences.

[20] Acknowledgments. The work at the University of Central Florida was supported by NASA grant NAG5-13507. BWR was supported by AF grant FA718-06-0072. The authors thank the reviewers for their comments and suggestions.

[21] Amitava Bhattacharjee thanks Stanley Solomon and another reviewer for their assistance in evaluating this paper.

\section{References}

Altadill, D., and E. M. Apostolov (2003), Time and scale size of planetary wave signatures in the ionospheric $\mathrm{F}$ region: Role of the geomagnetic activity and mesosphere/lower thermosphere winds, J. Geophys., 110, A01312.

Bailey, S. M., T. N. Woods, C. A. Barth, S. C. Solomon, R. Korde, and L. R. Canfield (2000), Measurements of the solar soft X-ray irradiance by the Student Nitric Oxide Explorer: First analysis and underflight calibrations, J. Geophys. Res., 105(A12), 27,179.

Bailey, S. M., T. N. Woods, F. G. Eparvier, and S. C. Solomon (2005), Observations of the solar soft X-ray irradiance by the Student Nitric Oxide Explorer, Adv. Space. Res., 37(2), 209-218.

Balan, N., G. J. Bailey, B. B. Jenkins, P. B. Rao, and R. J. Moffet (1994), Variations of ionospheric ionization and related solar irradiances during an intense solar cycle, J. Geophys. Res., 99(A2), 2243-2253.
Dodge, Y. (2006), The Oxford Dictionary of Statistical Terms, Oxford Univ. Press, New York.

Fagundes, P., R. Pillat, V. G. Bolzan, M. J. A. Sahai, Y. BeckerGuedes, F. Abalde, and J. R. Aranha (2005), Observations of F layer electron density profiles modulated by planetary wave type oscillations in the equatorial ionospheric anomaly region, J. Geophys. Res., 110, A12302, doi:10.1029/2005JA011115.

Forbes, J. M., S. E. Palo, and X. Zhang (2000), Variability of the ionosphere, J. Atmos. Sol. Terr. Phys., 62, 685-693.

Kane, K. P. (1992), Solar cycle variation of foF2, J. Atmos. Terr. Phys., 54 $1201-1205$

Lastovicka, J., P. Krizan, P. Sauli, and D. Novotna (2003), Persistence of the planetary wave type oscillations in foF2 over Europe, Ann. Geophys., $21,1543-1552$

Mendillo, M., H. Risbeth, R. G. Roble, and J. Wroten (2002), Modelling F2-layer seasonal trends and day-to-day variability driven by coupling with the lower atmosphere, J. Atmos. Sol.-Terr. Phys., 64, 1911-1931.

Mursula, K., and B. Zieger (1996), The 13.5 day periodicity in the Sun, solar wind, and geomagnetic activity: The last three solar cycles, J. Geophys. Res., 101, 27,077-27,090.

Pancheva, D. V, and N. J. Mitchell (2004), Planetary waves and variability of the semidiurnal tide in the mesosphere and lower thermosphere over Esrange $(68 \mathrm{~N}, 21 \mathrm{E})$ during winter, J. Geophys. Res., 109, A08307, doi:10.1029/2004JA010433.

Reinisch, B. W., and X. Huang (1983), Automatic calculation of electron density profiles from digital ionograms. 3. Processing of bottomside ionograms, Radio Sci., 18, 477.

Reinisch, B. W. (1996), Modern ionosondes, in Modern Ionospheric Science, edited by H. Kohl, R. Ruster, and K. Schlegel, 440-458, Eur. Geophys. Soc., 37191, Katlenburg-Lindau, Germany.

Rishbeth, H. (1993), Day-to-day ionospheric variations in a period of high solar activity, J. Atmos. Terr. Phys., 55, 165.

Rishbeth, H., and M. Mendillo (2001), Patterns of F2-layer variability, J. Atmos. Sol.-Terr. Phys., 63, 1661-1680.

Solomon, S. C., S. M. Bailey, and T. N. Woods (2001), Effect of solar soft X-rays on the lower ionosphere, Geophys. Res. Lett., 28(11), 2149-2152. Valladares, C. E., S. Basu, K. Groves, M. P. Hagan, D. Hysell, A. Mazzella, and R. Sheehan (2001), Measurements of equatorial spread-F ionospheric conditions using a latitudinal chain of GPS receivers, J. Geophys. Res., 106, 29133.

Wang, X., R. Eastes, S. Weichecki Vergara, S. Bailey, C. Valladares, and T. Woods (2006), On the short-term relationship between solar soft X-ray irradiances and equatorial total electron content (TEC), J. Geophys. Res., 111, A10S15, doi:10.1029/2005JA011488.

Woods, T., N. Eparvier, F. G. Bailey, S. M. Chamberlin, P. C. Gary, J. L. Rottman, J. Solomon, S. C. Tobiska, and W. K. Woodraskal (2005), Solar EUV Experiment (SEE): Mission overview and first results, J. Geophys. Res., 110, A01312, doi:10.1029/2004JA010765.

S. Bailey, Geophysical Institute, University of Alaska Fairbanks, Fairbanks, AK, USA

R. Eastes, Florida Space Institute, Kennedy Space Center, Merritt Island, FL, USA.

B. W. Reinisch, Center of Atmospheric Research, University of Massachusetts, Lowell, MA, USA.

C. E. Valladares, Newton Resource Center, Institute for Scientific Research, Boston College, Chestnut Hill, MA, USA.

$\mathrm{X}$. Wang, Department of Electrical and Computer Engineering, University of Central Florida, Orlando, FL, USA. (shannon_ucf@hotmail. com)

T. Woods, Laboratory for Atmospheric and Space Physics, University of Colorado, Boulder, CL, USA. 